\title{
Intact visual discrimination of complex and feature-ambiguous stimuli in the absence of perirhinal cortex
}

\author{
Daniel A. Levy, ${ }^{1,2}$ Yael Shrager, ${ }^{3}$ and Larry R. Squire ${ }^{1,2,3,4,5}$ \\ ${ }^{1}$ Veterans Affairs Healthcare System, San Diego, California 92161, USA; Departments of ${ }^{2}$ Psychiatry, ${ }^{3}$ Neurosciences, \\ and ${ }^{4}$ Psychology, University of California, San Diego, La Jolla, California 92093, USA
}

\begin{abstract}
The perirhinal cortex is known to be important for memory, but there has recently been interest in the possibility that it might also be involved in visual perceptual functions. In four experiments, we assessed visual discrimination ability and visual discrimination learning in severely amnesic patients with large medial temporal lobe lesions that included complete lesions of perirhinal cortex. Experiment 1 tested complex visual object perception. Experiments 2a and $2 b$ tested in two different ways the ability to discriminate between feature-ambiguous images, which was reported to be impaired in monkeys with perirhinal lesions. Experiment 3 involved images that were successfully discriminated in Experiment 2a and asked patients to learn across 20 trials which of the images had been designated as "correct." Patients performed as well as controls in Experiments $1,2 a$, and $2 b$, but one of the patients had difficulty in Experiment 3 when the task required remembering from trial to trial which image was "correct." These findings indicate that perirhinal cortex is not needed for visual perception across a wide range of visual perceptual tasks.
\end{abstract}

The perirhinal cortex is part of a system of medial temporal lobe structures important for declarative memory (Squire and ZolaMorgan 1991; Mishkin and Murray 1994). Because perirhinal cortex receives nearly two-thirds of its input from adjacent unimodal visual areas, including areas TE and TEO (Suzuki and Amaral 1994), there has been interest in the possibility that perirhinal cortex might be involved in visual perceptual functions in addition to memory functions.

Several studies have emphasized the contribution of perirhinal cortex to memory functions and have found little evidence for a role of perirhinal cortex in visual perception itself. Thus, monkeys with lesions of perirhinal cortex performed well at 0 -sec delays in the visual paired-comparison test, whereas lesions of area TE markedly impaired performance in both no-delay and delay tests (Buffalo et al. 1999). Patients with medial temporal lobe lesions that included perirhinal cortex were also impaired at delay tests, but not no-delay tests, of visual recognition memory (Buffalo et al. 1998; Holdstock et al. 2000). Monkeys with perirhinal lesions were able to generalize to altered views of previously learned stimuli but were impaired at initial learning (Hampton and Murray 2002). Similarly, severely amnesic patients with lesions that included the perirhinal cortex performed normally on seven different visual discrimination tasks (Stark and Squire 2000). Finally, the noted amnesic patient H.M., whose medial temporal lobe lesion includes most of the perirhinal cortex, performed normally on a number of visual perceptual tasks (Milner et al. 1968).

In contrast to these findings, recent studies of experimental animals have raised the suggestion that damage to perirhinal cortex impairs visual perception (Murray and Bussey 1999; Buckley et al. 2001; Bussey et al. 2003; Norman and Eacott 2004). For example, monkeys with lesions of perirhinal cortex were impaired at performing difficult visual discrimination tasks. When presented with five different orientations of one object and a

${ }^{5}$ Corresponding author.

E-mail Isquire@ucsd.edu; fax (858) 552-7457.

Article published online ahead of print. Article and publication date are at http://www.learnmem.org/cgi/doi/10.1101//m.84405. sixth orientation of a different object, monkeys with perirhinal lesions were impaired at choosing the odd object (Buckley et al. 2001).

It has also been suggested that the perirhinal cortex is specifically necessary for visual discriminations between stimuli having high feature ambiguity (Bussey et al. 2003; Norman and Eacott 2004). Feature ambiguity occurs when particular features of an object are rewarded when they are part of one object but not when part of another object (Murray and Bussey 1999; Bussey and Saksida 2002; Bussey et al. 2003). Monkeys with lesions of perirhinal cortex were impaired at learning to discriminate between two images and, after learning to discriminate, were further impaired at discriminating feature-ambiguous stimuli that were created by blending the two images so that they contained overlapping features (Bussey et al. 2003).

In studies of visual perception in monkeys, it is difficult to distinguish impairments in visual perception from impairments in learning and memory. In contrast, this distinction can be made rather easily in studies of patients, who can be instructed about the nature of the task. We have explored visual discrimination abilities and visual discrimination learning in severely amnesic patients with medial temporal lobe lesions, including complete lesions of perirhinal cortex. Experiment 1 assessed the capacity for making difficult visual discriminations among six faces, as had been tested in monkeys (Buckley et al. 2001). In our earlier study of this same task (Stark and Squire 2000), the patients performed well overall, but two of the three patients performed numerically below the poorest control subject on this one test. Experiments $2 \mathrm{a}$ and $2 \mathrm{~b}$ tested the ability of patients to discriminate between blended (feature-ambiguous) images, using the same kind of stimulus materials that were used in work with monkeys (Bussey et al. 2003). Experiment 2a asked patients to make same-different judgments for pairs of blended images. Three different levels of blending were used, including two of the levels that had been used in the study of monkeys. Experiment $2 \mathrm{~b}$ asked patients to inspect an image and to decide which of two blended images was most similar to it. Experiment 3 presented pairs of images that had been successfully discriminated in Ex- 
periment $2 \mathrm{a}$ and asked patients to learn which image had been designated "correct" and which had been designated "incorrect." In this way, we compared the ability to discriminate between two highly similar images (Experiments $2 \mathrm{a}$ and $2 \mathrm{~b}$ ) and the ability to learn and remember across several trials which image was correct (Experiment 3).

\section{Results}

\section{Experiment 1}

Figure 1 shows percentage correct scores for each of the two patients and their controls on three different face discrimination tests. E.P.'s mean score $(54.0 \%$ correct; chance performance $=16.7 \%$ ) was numerically poorer than the mean score obtained by his four controls $(69.1 \%$ correct $\pm 4.2 \%)$ but was within the 95\% confidence interval of their scores (51.1\%). G.P.'s performance $(77.3 \%$ correct) was a little better than the mean score obtained by his four controls $(72.0 \%$ correct $\pm 3.8 \%)$. This finding indicates that patients with large medial temporal lobe lesions, including complete perirhinal damage, can perform well on a difficult visual discrimination task. G.P. performed entirely normally, and E.P. performed normally on one occasion and a little worse than controls on two other occasions.

\section{Experiment 2a}

Figure 2A shows percent correct scores for the discrimination of feature-ambiguous pictures. All participants performed with $100 \%$ accuracy in the "Easy" condition. In the "Hard" condition, both patients scored $100 \%$ correct (controls, $97.9 \% \pm 0.7 \%$ correct). In the "Very Hard" condition, G.P. scored 100\% correct, E.P. scored $83.3 \%$ correct, and the controls scored $87.5 \% \pm 2.0 \%$ correct (range $=75.0 \%-91.7 \%$; E.P.'s score was within the $95 \%$ confidence interval of the control scores, $82.2 \%$ ). In the "Same" condition, E.P. scored $96.0 \%$ correct, G.P. scored $87.5 \%$ correct, and controls scored $96.9 \% \pm 0.9 \%$ correct. Finally, the total percent correct scores across all 96 trials were similar for the patients and the controls (G.P., 96.9\%; E.P., 94.8\%, controls, $95.6 \% \pm 0.5 \%)$.

G.P.'s performance on the "Same" condition indicated that he had a bias for labeling image pairs as different. Accordingly, we also calculated a discriminability measure $\left(\mathrm{d}^{\prime}\right)$, using the method developed for same-different judgments (MacMillan and Creelman 1991). In this analysis, correct responses of "different" to images that were in fact different were designated as Hits, and incorrect responses of "different" to images that were in fact the same were designated as False Alarms. Scores of 1.0 or 0.0 for Hits and False Alarms were subjected to a standard correction (Hits $=1-1 /(2 n)$; False Alarms $=0+1 /(2 n)$ ). Figure 2B shows

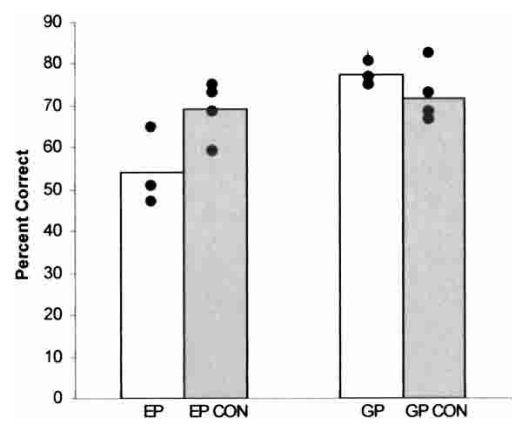

Figure 1. Performance by E.P., G.P., and controls $(\mathrm{CON}, n=4)$ on three tests of the ability to discriminate faces (Experiment 1). Black circles show patient scores on each of the three tests and the mean of each control's scores. Chance $=16.7 \%$ correct. that the patients and controls performed similarly (E.P., $\mathrm{d}^{\prime}=5.10$; G.P., $\mathrm{d}^{\prime}=5.49$; controls, $\mathrm{d}^{\prime}=5.38 \pm 0.13$ ). On this measure, G.P. performed better than most controls, and E.P.'s score was within the control range (lowest score: 4.67$)$ and within the $95 \%$ confidence interval of the control scores (5.07). The False Alarm rate was 0.12 for G.P., 0.04 for E.P., and 0.03 for controls (range $=0-0.08$ ). The results were virtually the same when Hits were defined as correct responses to images that were the same and False Alarms were defined as incorrect responses to images that were different.

\section{Experiment $2 b$}

All participants were able to judge which blended image was more similar to the central image. Of the controls $(n=4)$, two scored $100 \%$, one scored 99\%, and one scored 94\%. G.P. scored $99 \%$, and E.P. scored $100 \%$. When deciding which of the two blended images was the correct choice, both G.P. and E.P. indicated in their remarks that they perceived the images at the level of complex objects. For example, on one occasion, when explaining his decision, G.P. said, "The woman with the wheelbarrow is clearer in this one." On another occasion, E.P. said, "The clock is more visible in this one."

\section{Experiment 3}

All participants readily learned the discrimination problem that was based on two blended images from the "Hard" condition in Experiment 2a. The controls $(n=4)$ and G.P. scored 100\% correct, and E.P. scored 95\% correct. E.P. chose incorrectly on the fourth trial of testing and responded correctly on the other trials. In the "Very Hard" condition, all four controls and G.P. performed perfectly after the first trial. In contrast, E.P. had difficulty learning some of the discrimination problems. Thus, for the five discrimination problems on which he was tested, E.P. learned two problems successfully, scoring $100 \%$ correct on trials 11-20. However, he failed to learn the other three problems $(50 \%$ correct on trials 11-20). E.P. performed poorly despite the fact that he was able to discriminate between the images that he was trying to learn and was able for each problem to point out differences between them.

\section{Discussion}

Two patients with large medial temporal lobe lesions and severe amnesia were given tests of visual discrimination and visual discrimination learning. In Experiment 1, the patients saw five orientations of one face and a sixth orientation of another face. The task was to select the odd face. E.P. scored within the 95\% confidence interval of control scores, and G.P. performed slightly better than controls. Experiment 2a asked the patients to judge whether pairs of blended images were exactly the same or different. Experiment $2 \mathrm{~b}$ asked patients to determine which of two blended images was more similar to a given distinct image. E.P. and G.P. performed normally. Experiment 3 presented two blended images that had been successfully discriminated in Experiment 2 a. Patients attempted to learn across 20 trials which image was designated as "correct." E.P. and G.P. learned quickly to discriminate image pairs that came from the "Hard" condition of Experiment $2 \mathrm{a}$ and performed as well as controls. For image pairs that came from the "Very Hard" condition, G.P. learned as quickly as controls, but E.P. was impaired.

These findings show that complex visual discrimination performance and discrimination between images having high feature ambiguity can succeed despite complete bilateral lesions of perirhinal cortex. Impaired performance occurred only during tests of discrimination learning.

\section{Learning \& Memory}



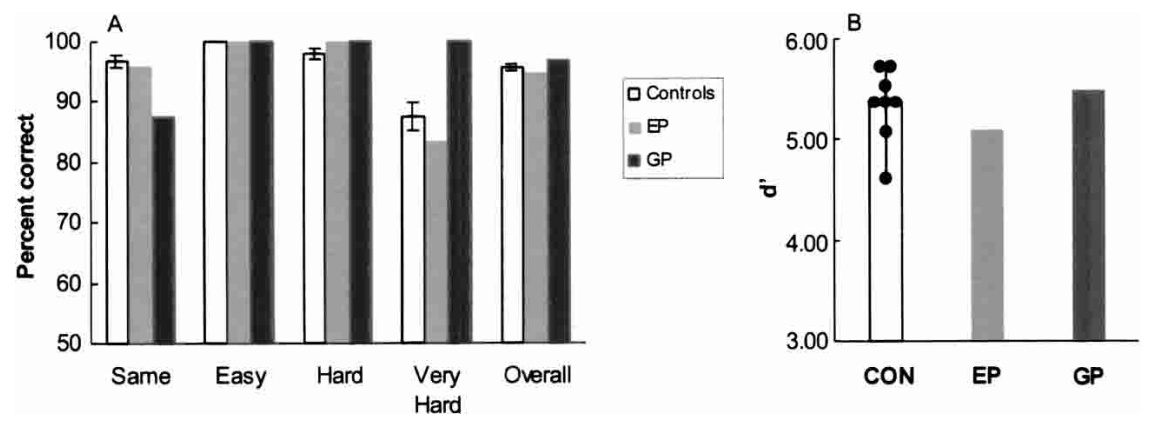

Figure 2. (A) Percent correct scores for amnesic patients with perirhinal lesions (E.P. and G.P.) and controls (CON, $n=8$ ) who made same-different judgments for pairs of blended images (Experiment 2a). Scores are shown separately for each of four conditions (Same, Easy, Hard, and Very Hard) as well as for all 96 image pairs. Brackets indicate std. error of the mean. (B) Discrimination accuracy $\left(d^{\prime}\right)$ scores for patients and controls for all trial types. Hit $=$ correct response for one of the 72 "different" image pairs. False Alarm = incorrect response for one of the 24 "Same" image pairs. Black circles show scores of the eight controls.

In accordance with the present findings, Hampton and Murray (2002) reported that lesions of the perirhinal cortex impaired visual learning but not visual perception. Monkeys were first trained to discriminate between pairs of visual stimuli (displays of designs, outline drawings, and objects). Then, while testing continued, the monkeys were given interpolated probe trials to assess the ability to discriminate when the stimuli were manipulated. Monkeys with lesions of perirhinal cortex were slow to learn the original discriminations but then performed as well as controls when the stimuli were rotated $\left(30^{\circ}-120^{\circ}\right)$, enlarged, shrunk, presented with color removed, or degraded with masks. Thus, in the absence of perirhinal cortex, monkeys exhibited intact perception as measured by generalization to altered views of familiar stimuli.

Other studies have reached a different conclusion, namely, that perirhinal cortex is critical for the processing of visual information, including the ability to identify complex objects (Buckley and Gaffan 1998; Buckley et al. 2001; Norman and Eacott 2004). Thus, monkeys with lesions of the perirhinal cortex were impaired at learning to make difficult visual discriminations (Buckley et al. 2001), including the odd-face discrimination task in Experiment 1 of the present study. Yet, E.P. and G.P. performed well on the odd-face discrimination task. One reason for this discrepancy might be that the lesions in the monkey inadvertently extended into the laterally adjacent area TE, as discussed previously (Buffalo et al. 1999). A second reason for the discrepancy might rest on the fact that it is difficult with experimental animals to distinguish between an impaired ability to identify objects and an impaired ability to learn which of two objects is the correct one (Buffalo et al. 1999; Bussey et al. 2003). A third possibility is that there are substantive species differences between monkeys and humans with respect to the function of anterior temporal lobe structures (also see Stark and Squire 2000).

Bussey et al. (2003) advanced a somewhat different proposal that the perirhinal cortex is important for discriminating between objects that have a high degree of feature ambiguity. Monkeys first learned to discriminate between pairs of distinct images (Low Feature Ambiguity). Monkeys with lesions of the perirhinal cortex took $36.4 \pm 6.5$ trials to reach criterion, and control monkeys took $24.8 \pm 3.6$ trials to reach criterion. The monkeys were then given blocks of 32 test trials involving blends of the same two images. One blending condition corresponded to the "Easy" condition in our Experiments $2 \mathrm{a}$ and $2 \mathrm{~b}$, and the other blending condition corresponded to the "Very Hard" condition in Experiments $2 \mathrm{a}$ and $2 \mathrm{~b}$. The finding was that monkeys with perirhinal lesions were impaired at learning the initial discrimination and were also impaired during the subsequent test trials involving the blended images. In this respect, the results differed from the earlier report that monkeys with perirhinal lesions could generalize successfully to altered stimuli after they completed discrimination training (Hampton and Murray 2002). In the case of the study with blended images, it was suggested that learning was required during initial discrimination learning but not during the 32 test trials with blended images. Accordingly, it was suggested that impaired performance during the trials with blended images reflected impaired visual perception, not an impairment in learning and memory.

This interpretation was based on an analysis of variance that indicated that no further learning occurred across the 32 trials (the 32 trials were partitioned into eight four-trial blocks). There was also no group $\times$ trial-block interaction. Yet, one wonders how monkeys performed on the first one or two blocks of the test trials with blended images. Did generalization fail altogether? One also wonders whether the impaired performance during these test trials could reflect to some degree the fact that control monkeys improved more during the test trials than the monkeys with perirhinal lesions. Did no learning occur at all? Accordingly, it would be of interest to inspect the performance curves across the blocks of trials with blended images to determine whether there was any sign of differential learning.

A recent study of rats also illustrates the difficulty in distinguishing between impaired memory for objects and impaired perception of objects (Norman and Eacott 2004). Rats with lesions of perirhinal cortex did not exhibit the normal tendency to explore a novel object rather than a familiar object, when the novel object was a reconfigured version of the familiar object rather than a completely different object. This finding was interpreted as supporting the idea that the perirhinal cortex is critical for discriminating between feature-ambiguous stimuli. Yet, the two test objects were presented after delays ranging from $1 \mathrm{~min}$ to $24 \mathrm{~h}$. To decide whether perception was impaired, rather than delay-dependent memory, it would have been important to test animals at no delay. This strategy has been successful previously in distinguishing the visual perceptual functions of area TE from the memory functions of perirhinal cortex. Monkeys with TE lesions were impaired at a 0-sec delay on a test of novel object recognition, whereas monkeys with perirhinal lesions were intact at a 0-sec delay and impaired at longer delays (Buffalo et al. 1999).

In our study, we isolated the process of making visual discriminations from the processes of learning and memory by eliminating the need to learn a correct response or to retain stimulus-reward associations in memory (Experiments 1, 2a, and 2b). Patients E.P. and G.P. performed well in these tasks. In Experiment 3 , we introduced a learning requirement by asking participants to learn that one image in a pair was "correct" and that the other was "incorrect." E.P. and G.P. performed well when the difference between the two images was straightforward (the "Hard" condition). G.P. also performed well in the "Very Hard" condition. Patient E.P. was impaired in this condition on three of five discrimination problems, even though he could correctly point out ways in which the two blended images were different. It appeared that, because the images were quite similar in appearance, E.P. had difficulty remembering from trial to trial which of the images was the correct one. E.P. likely succeeded in the sim- 
pler "Hard" condition because he could readily discriminate between the images and maintain a visual representation or a verbal label in working memory. We suggest that E.P. was impaired at learning some of the "Very Hard" discrimination problems because his performance is especially dependent on explicit rehearsal and maintenance of working memory. These abilities, which are intact in E.P., may on their own be insufficient to support a high level of performance. Thus, controls could have benefited not only from the ability to rehearse information in working memory but also from the ability to recollect incidental information about the images in the absence of explicit rehearsal.

We found that visual perceptual performance was intact after perirhinal lesions when we eliminated altogether the need to learn and remember. The present findings therefore suggest that the perirhinal cortex is not necessary for making difficult visual discriminations, including discriminations between stimuli with a high degree of feature ambiguity.

\section{Materials and Methods}

\section{Experiment 1}

\section{Participants}

We tested two male patients who have extensive medial temporal lobe lesions and severe amnesia as a result of herpes simplex encephalitis (Fig. 3). E.P. was born in 1922, had 12 yr of education, and developed amnesia in 1992. G.P. was born in 1946, had $16 \mathrm{yr}$ of education, and developed amnesia in 1987. Both patients have severe amnesia. Neither patient could recall any of a short prose passage after a 12 -min delay ( 0 segments correct). Neither patient showed evidence of paired associate learning [0, 0 , and 0 pairs recalled on three successive trials (maximum score $=10 /$ trial)]. For the Rey-Osterieth figure, E.P. obtained a score for copying the figure of 27 and a delayed (12-min) reproduction score of 0 . G.P. obtained a copy score of 25.5 and a reproduction score of 4 (average of two tests).

Estimates of medial temporal lobe damage were based on quantitative analysis of magnetic resonance images from the patients and four controls for each patient, following published procedures for segmenting the temporal lobe (Insausti et al. 1998; Amaral and Insausti 1990; also see Gold and Squire 2005). Volume estimates for the lateral temporal lobe, frontal lobe, parietal lobe, and occipital lobe were also obtained. The measurements presented here are based on improved techniques and on
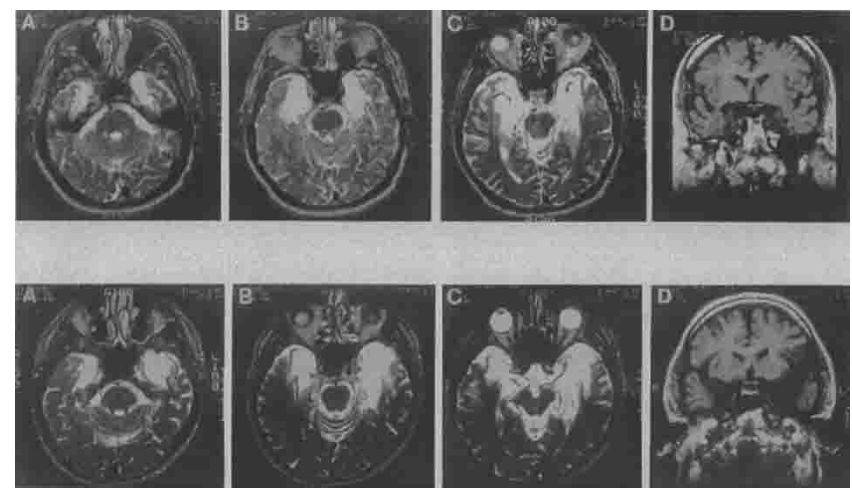

Figure 3. Magnetic resonance images showing the extent of temporal lobe damage in patients E.P. (top row) and G.P. (bottom row). ( $A-C$ ) In each row are T-2 weighted axial images through the temporal lobe. The images are continuous $5-\mathrm{mm}$ sections (with $2.5-\mathrm{mm}$ gaps) and are arranged from ventral $(A)$ to dorsal $(C)$. Damaged tissue is indicated by bright signal. $D$ in each row is a coronal T-1 weighted image at the level of the amygdala. Damaged tissue is indicated by dark signal. See text for description of the lesions. more controls than were available previously. Accordingly, the estimates of damage are similar but not identical to what was earlier reported for these two patients (Schmolck et al. 2002; Levy et al. 2004).

E.P.'s lesion extends $7 \mathrm{~cm}$ caudally from the temporal pole bilaterally and includes all of the amygdala and all of the hippocampal region (dentate gyrus, cell fields of the hippocampus proper, and subicular complex, except for a small tag of abnormally appearing vestigial tissue that comprises $\sim 3 \%$ of hippocampal volume). In addition, the damage includes all of the entorhinal cortex, all of the perirhinal cortex, and much of the parahippocampal cortex ( $70 \%$ on the left and $76 \%$ on the right). The lesion also extends laterally to include the rostral portion of the fusiform gyrus $(-39 \%$ on the left and $68 \%$ on the right). The insula is reduced in size ( $\sim 32 \%$ on the left and $30 \%$ on the right). Lastly, the lateral temporal lobe (inferior, middle, and superior temporal gyri) is of normal size bilaterally (within $7 \%$ of the mean control volume), as are the frontal, parietal, and occipital lobes (all $\pm 9 \%$ of the mean control volumes).

G.P.'s lesion is primarily medial temporal, but his lesion extends further laterally than E.P.'s lesion. Damage extends through the anterior $7 \mathrm{~cm}$ of the left temporal lobe and the anterior $6 \mathrm{~cm}$ of the right temporal lobe. The damage includes bilaterally all of the amygdala, all of the hippocampal region (except $7 \%$ on the right), all of the entorhinal and perirhinal cortices, and much of the parahippocampal cortex $(\sim 87 \%$ on the left and $55 \%$ on the right). The damage also includes the anterior $1 \mathrm{~cm}$ of the temporal lobe and extends into the fusiform gyrus ( $\sim 41 \%$ on the left and $56 \%$ on the right). The insula is reduced in volume by $\sim 80 \%$ on the left and $49 \%$ on the right. Lastly, the lateral temporal lobe (inferior, middle, and superior temporal gyri) is within $13 \%$ of the mean control volume. The volumes of the frontal, parietal, and occipital cortices are all within $\pm 10 \%$ of the mean control volumes.

Eight healthy males served as controls for the amnesic patients. Four were matched to E.P. (mean age $=74.3 \mathrm{yr}, 13.5 \mathrm{yr}$ of education), and four were matched to G.P. (mean age $=56.8 \mathrm{yr}$, $12.3 \mathrm{yr}$ of education).

\section{Materials and procedure}

Ten male human faces were used, each photographed in black and white from six different orientations. On each of 40 trials, five different orientations of one face and a sixth orientation of another face were presented on a computer screen in a $2 \times 3$ display. The odd face was randomly selected, as was the orientation of the odd face and the location of the odd face in the $2 \times 3$ display. Each trial involved a unique display (for a sample trial, see Stark and Squire 2000). Trials were self-paced, and participants pressed one of six keys in a $2 \times 3$ array that matched the computer display in order to indicate which of the six faces was different from the other five. Three different tests were administered on different days to each participant.

\section{Experiment 2a}

\section{Participants}

The same patients (E.P. and G.P.) who participated in Experiment 1 also participated in Experiment 2a. Eight healthy males (two of whom also participated in Experiment 1) served as controls for the amnesic patients. Four were matched to E.P. (mean age $=80$ $\mathrm{yr}, 11.6 \mathrm{yr}$ of education), and four were matched to G.P. (mean age $=62.3 \mathrm{yr}, 15.2 \mathrm{yr}$ of education). Because these two groups performed similarly (the older participants scored marginally better), their scores were combined.

\section{Materials and procedure}

The test stimuli were 96 pairs of gray-scale images. Each image in a pair was a blend of two different digital photographs (Fig. 4) prepared with the freeware program WinMorph 3.01 (DebugMode, www.debugmode.com). To create the test material, pairs of digital photographs were used to create a series of 40 different blends. Images at one end of the series were more similar to one

\section{Learning \& Memory}




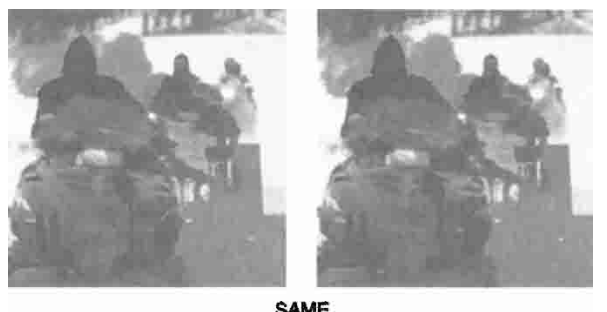

SAME
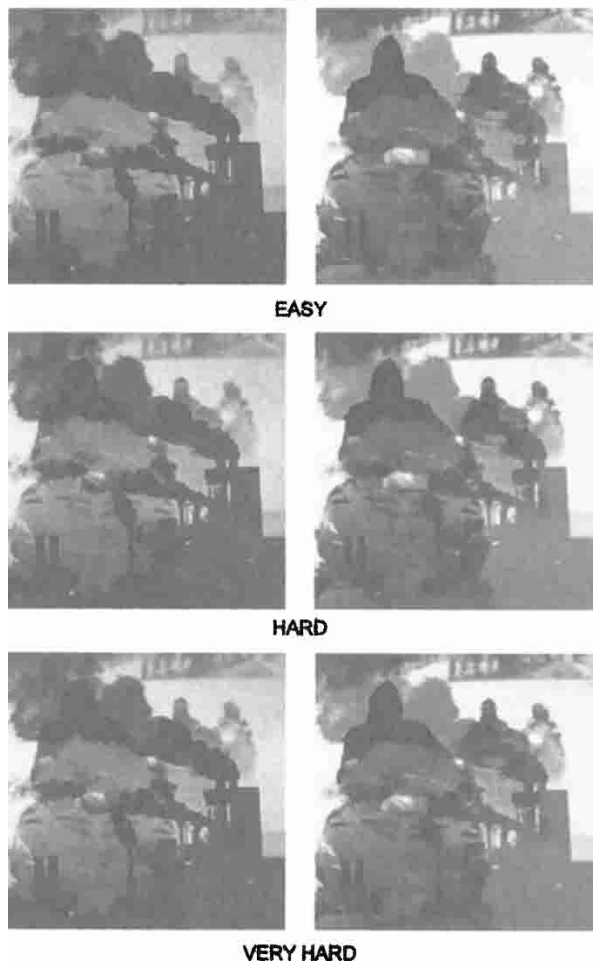

Figure 4. Examples of blended image pairs (Experiment 2a). Participants were asked to judge whether pairs of images were exactly the same or different. Twenty-four image pairs were presented in each of four conditions (Same, Easy, Hard, Very Hard). In the test itself, each of the 96 image pairs was derived from a unique pair of photographs. Thus, only one of the image pairs illustrated here actually appeared in the test.

of the two photographs, images at the other end of the series were more similar to the other photograph, and images in the middle of the series were nearly equal blends of both photographs. The progression through the 40 steps was made by altering the monochromatic strength of individual pixels. This procedure was then followed for a total of 96 different pairs of source photographs, resulting in a graded series of 40 blended images from each of the 96 pairs. One pair of images was then selected from each series to create 24 test pairs in each of four different conditions (Same, Easy, Hard, Very Hard).

In the "Same" condition, the two images were identical and were taken from either step 25 or step 27 along the 40-step blending continuum. In the "Easy" condition, one image was from step 14 , and the other image was from step 27 (12 steps of separation). This degree of difficulty was identified as "High Feature Ambiguity" in the study with monkeys by Bussey et al. (2003) (Experiment 1). In the "Hard" condition, one image was from step 16, and the other was from step 25 (eight steps of separation). In the "Very Hard" condition, one image was from step 17, and the other image was from step 24 (six steps of separation). This degree of difficulty was employed as an additional "high feature ambiguity" condition by Bussey et al. (2003) (Experiment 2).

On each trial, participants saw two images displayed on a computer screen side by side (each image was $\sim 9 \times 9 \mathrm{~cm}$ ). Participants were asked to examine each pair of images without time constraint and press one of two keys to indicate whether the two images were "exactly the same" or whether they were "not the same." Prior to testing, a practice set of eight image pairs was presented with feedback, and participants were instructed that the difference between the two images could be quite subtle and that the images should be identified as different even if they were only slightly different. The test session itself consisted of 96 trials, mixed with respect to difficulty. No feedback was given during the test.

\section{Experiment $2 b$}

\section{Participants}

The same patients (E.P. and G.P.) who participated in Experiments 1 and $2 \mathrm{a}$ also participated in Experiment $2 \mathrm{~b}$. Four healthy males served as controls for the amnesic patients. Two were matched to E.P. (mean age $=81.5 \mathrm{yr}, 12 \mathrm{yr}$ of education), and two were matched to G.P. (mean age $=61.5 \mathrm{yr}, 13 \mathrm{yr}$ of education). Because these two groups performed similarly (the younger participants scored marginally better), their scores were combined.

\section{Materials and procedure}

The test stimuli were 72 pairs of blended images from Experiment $2 \mathrm{a}$, together with the original images from which the blended images were derived (Fig. 5). Thirty-six image pairs were presented in the "Hard" condition, and 36 pairs were presented in the "Very Hard" condition. On each trial, three images were displayed in a row on the computer screen. The middle image was one of the two original images that had been blended to create the left and right images. Participants were asked to decide whether the left image or the right image was more similar to the middle image. Prior to testing, a practice set of 10 trials was presented with feedback.

\section{Experiment 3}

\section{Participants}

The same patients (E.P. and G.P.) who participated in Experiments 1, 2a, and 2b also participated in Experiment 3. Four healthy males who participated in Experiment 2a (two matched to E.P. and two matched to G.P.) served as controls for the patients.

\section{Materials and procedure}

Visual discrimination learning was assessed with two different tasks. The first task involved a single pair of images that all participants had successfully discriminated in the "Hard" condition in Experiment 2a. The second task involved pairs of images that
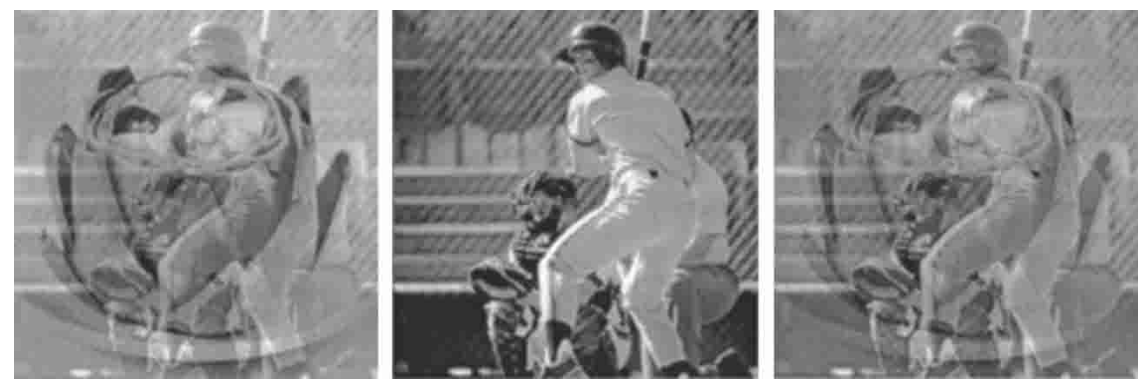

Figure 5. Example of the "Very Hard" condition in Experiment 3. Participants were asked to judge which blended image was most similar to the middle image. Thirty-six trials were presented in each of two conditions (Hard and Very Hard). 
all participants had successfully discriminated in the "Very Hard" condition in Experiment 2a. In both tasks, a pair of images was presented on the computer screen. Participants were told that one image was correct and the other incorrect and that they should indicate by key press which image they believed to be the correct one. Feedback (correct, incorrect) was given after each of 20 trials. For the first task ("Hard" condition), which proved to be quite easy, 20 learning trials were presented. For the second task ("Very Hard" condition), 20 trials were also given. In this condition, controls were tested on one occasion, G.P. was tested on two different occasions with two different pairs of images, and E.P. was tested on five different occasions with five different pairs of images.

\section{Acknowledgments}

Supported by the Medical Research Service of the Department of Veterans Affairs, NIMH Grant 24600, NIA Grant AG05131 to the Alzheimer's Disease Research Center at UCSD, and the Metropolitan Life Foundation. Timothy Bussey graciously provided stimuli. We thank John Wixted for discussion, Jennifer Frascino and Leah Swalley for research assistance, and Jeffrey Gold and Peter Bayley for analysis of magnetic resonance images.

\section{References}

Amaral, D.G. and Insausti, R. 1990. The hippocampal formation. In The human nervous system (ed. G. Paxinos), pp. 711-755. Academic Press, San Diego, CA.

Buckley, M.J. and Gaffan, D. 1998. Perirhinal cortex ablation impairs visual object identification. J. Neurosci. 18: 2268-2275.

Buckley, M.J., Booth, M.C., Rolls, E.T., and Gaffan, D. 2001. Selective perceptual impairments after perirhinal cortex ablation. J. Neurosci. 21: 9824-9836.

Buffalo, E.A., Reber, P.J., and Squire, L.R. 1998. The human perirhinal cortex and recognition memory. Hippocampus 8: 330-339.

Buffalo, E.A., Ramus, S.J., Clark, R.E., Teng, E., Squire, L.R., and Zola S.M. 1999. Dissociation between the effects of damage to perirhinal cortex and area TE. Learn. Mem. 6: 572-599.

Bussey, T.J. and Saksida, L.M. 2002. The organization of visual object representations: A connectionist model of effects of lesions in perirhinal cortex. Eur. J. Neurosci. 15: 355-364.

Bussey, T.J., Saksida, L.M., and Murray, E.A. 2003. Impairments in visual discrimination after perirhinal cortex lesions: Testing 'declarative' vs. 'perceptual-mnemonic' views of perirhinal cortex function. Eur. J. Neurosci. 17: 649-660.

Gold, J.J. and Squire, L.R. 2005. Quantifying medial temporal lobe damage in memory-impaired patients. Hippocampus (in press).

Hampton, R.R. and Murray, E.A. 2002. Stimulus representations in rhesus monkeys with perirhinal cortex lesions. Behav. Neurosci. 116: $363-377$.

Holdstock, J.S., Gutnikov, S.A., Gaffan, D., and Mayes, A.R. 2000. Perceptual and mnemonic matching-to-sample in humans: Contributions of the hippocampus, perirhinal and other medial temporal lobe cortices. Cortex 36: 301-322.

Insausti, R., Juottonen, K., Soininen, H., Insausti, A.M., Partanen, K., Vainio, P., Laakso, M.P., and Pitkanen, A. 1998. MR volumetric analysis of the human entorhinal, perirhinal, and temporopolar cortices. Am. J. Neuroradiol. 19: 659-671.

Levy, D.A., Bayley, P.J., and Squire, L.R. 2004. The anatomy of semantic knowledge: Medial vs. lateral temporal lobe. Proc. Natl. Acad. Sci. 101: 6710-6715.

MacMillan, N.A. and Creelman, C.C. 1991. Signal detection theory: A user's guide. Cambridge University Press, New York.

Milner, B., Corkin S., and Teuber, H.L. 1968. Further analysis of the hippocampal amnesic syndrome: 14 year follow-up study of H.M. Neuropsychologia 6: 215-234.

Mishkin, M. and Murray, E.A. 1994. Stimulus recognition. Curr. Opin. Neurobiol. 4: 200-206.

Murray, E.A. and Bussey, T.J. 1999. Perceptual-mnemonic functions of perirhinal cortex. Trends Cogn. Sci. 3: 142-151.

Norman, G. and Eacott, M.J. 2004. Impaired object recognition with increasing levels of feature ambiguity in rats with perirhinal cortex lesions. Behav. Brain Res. 148: 79-91.

Schmolck, H., Kensinger, E.A., Corkin, S., and Squire, L.R. 2002. Semantic knowledge in patient H.M. and other patients with bilateral medial and lateral temporal lobe lesions. Hippocampus 12: $520-533$.

Squire, L.R. and Zola-Morgan, S. 1991. The medial temporal lobe memory system. Science 253: 1380-1386.

Stark, C.E.L. and Squire, L.R. 2000. Intact visual perceptual discrimination in humans in the absence of perirhinal cortex. Learn. Mem. 7: 273-278.

Suzuki, W.A. and Amaral, D.G. 1994. Perirhinal and parahippocampal cortices of the macaque monkey: Cortical afferents. J. Comp. Neurol. 350: $497-533$.

Received July 22, 2004; accepted in revised form November 3, 2004.

\section{Learning \& Memory}




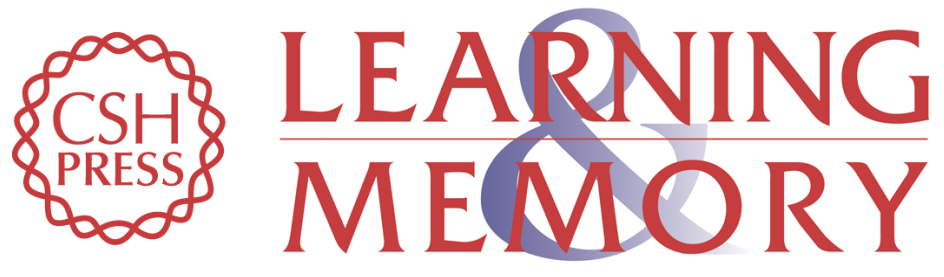

\section{Intact visual discrimination of complex and feature-ambiguous stimuli in the absence of perirhinal cortex}

Daniel A. Levy, Yael Shrager and Larry R. Squire

Learn. Mem. 2005, 12:

Access the most recent version at doi:10.1101/lm.84405

References This article cites 18 articles, 6 of which can be accessed free at: http://learnmem.cshlp.org/content/12/1/61.full.html\#ref-list-1

License

Email Alerting Receive free email alerts when new articles cite this article - sign up in the box at the Service top right corner of the article or click here. 